\title{
The interaction of DIAP1 with dOmi/HtrA2 regulates cell death in Drosophila
}

\author{
FS Khan ${ }^{1,2}$, M Fujioka ${ }^{1,2}$, P Datta $^{1,2}$, T Fernandes-Alnemri ${ }^{1}$, JB Jaynes $^{1}$ and ES Alnemri ${ }^{\star 1}$
}

Mitochondrial proteins such as cytochrome $c$, Smac/DIABLO and Omi/HtrA2 play important roles in the cell death pathways of mammalian cells. In Drosophila, the role of mitochondria in cell death is less clear. Here, we report the identification and characterization of the Drosophila ortholog of human Omi/HtrA2. We show that Drosophila $0 \mathrm{mi} / \mathrm{HtrA2}$ is imported into the mitochondria where it undergoes proteolytic maturation to yield two isoforms, dOmi-L and dOmi-S. dOmi-L contains a canonical $\mathrm{N}$-terminal IAP-binding motif (AVVS), whereas dOmi-S contains a distinct N-terminal motif (SKMT). DIAP1 was able to bind to both isoforms via its BIR1 and BIR2 domains. This resulted in cleavage of the linker region of DIAP1 between the BIR1 and BIR2 domains and further degradation of the BIR1 domain by the proteolytic activity of dOmi. The binding of DIAP1 to dOmi also resulted in DIAP1-mediated polyubiquitination of dOmi, suggesting that DIAP1 could target dOmi for proteasomal degradation. Consistent with this, expression of DIAP1 in Drosophila eye discs protected them from dOmi-induced eye ablation, indicating that DIAP1 plays an important role in protecting cells from the potentially lethal effects of dOmi. The ability of IAPs to bind to and ubiquitinate mitochondrial proteins such as dOmi may be a key conserved function to counterbalance the lethal effects of these proteins if accidentally released into the cytosol.

Cell Death and Differentiation (2008) 15, 1073-1083; doi:10.1038/cdd.2008.19; published online 15 February 2008

Like many higher organisms, Drosophila melanogaster makes extensive use of controlled cell death or apoptosis during development, morphogenesis, and homeostasis, to sculpt and maintain various tissues and organs. In mammals, the abundance of experimental evidence showing the important role of mitochondria in the intrinsic cell death pathway is compelling. ${ }^{1}$ However, in Drosophila the role and mechanism of action of mitochondrial proteins during intrinsic cell death remains obscure ${ }^{2}$ despite the reporting of altered cytochrome $c$ display in flies immediately prior to cell death, ${ }^{3}$ and the presence of two Bcl-2 (B-cell lymphoma 2)-like proteins, Debcl/dBorg1/dRob1 (pro-apoptotic) and dBorg2/ Buffy (antiapoptotic). ${ }^{4}$ Nevertheless, a recent report suggests that mitochondrial disruption plays an important role in Drosophila apoptosis. ${ }^{5}$

In Drosophila, several antagonists of inhibitor of apoptosis proteins (IAPs), Reaper, Grim, Hid, Sickle, and Jafrac-2, referred to collectively as IAP antagonists, have been characterized and shown to play important roles in cell death. ${ }^{6-10}$ In mammals, only two antagonists of IAPS, Smac (second mitochondrial-derived activator)/DIABLO (direct IAPbinding protein with low $\mathrm{pl}$ ) and Omi/HtrA2, have been well characterized. ${ }^{11}$ Unlike the Drosophila IAP antagonists, which are predominantly located in the cytosol, Smac/DIABLO and $\mathrm{Omi} / \mathrm{HtrA} 2$ are mitochondrial proteins located in the mitochondrial intermembrane space. The only structural or sequence homology between the Drosophila and mammalian IAP antagonists is a tetrapeptide at the $\mathrm{N}$ terminus, which constitutes a canonical IAP-binding motif (IBM). ${ }^{12}$ In Drosophila cells that are destined to die, the antiapoptotic function of Drosophila IAP1 (DIAP1) is thwarted by the IAP antagonists. $^{12}$ The IAP antagonists promote cell death by disrupting DIAP1-caspase association and/or by inducing DIAP1's auto-ubiquitination and degradation by the proteasomal pathway, thereby alleviating its inhibition of caspases. ${ }^{6,10,13-19}$

Several recent studies have demonstrated that mammalian $\mathrm{Omi} / \mathrm{HtrA} 2$ is a serine protease that is synthesized as a precursor, translocates to the mitochondrial intermembrane space, and there it is processed to its mature form by proteolytic cleavage, resulting in exposure of its IBM. ${ }^{20-23}$ Upon an apoptotic stimulus, Omi/HtrA2 is released into the cytosol, where it promotes caspase-dependent apoptosis, in part by mechanisms similar to those attributed to Smac/DIABLO, ${ }^{24,25}$ by preventing XIAP (X-linked inhibitor of apoptosis protein) from binding to caspases, or by disrupting already formed XIAP-caspase complexes. In addition,

\footnotetext{
${ }^{1}$ Department of Biochemistry and Molecular Biology, Center for Apoptosis Research, Kimmel Cancer Institute, Thomas Jefferson University, Philadelphia, PA, USA ${ }^{*}$ Corresponding author: ES Alnemri, Department of Biochemistry and Molecular Biology, Center for Apoptosis Research, Kimmel Cancer Institute, Thomas Jefferson University, 233 S10th Street, Philadelphia, PA 19107, USA. Tel: + 215503 4632; Fax: + 215923 1098; E-mail: e_alnemri@mail.jci.tju.edu

${ }^{2}$ These authors contributed equally to this work.

Keywords: Omi; HtrA2; Reaper; DIAP1; apoptosis; Drosophila

Abbreviations: BIR, baculovirus inhibitor of apoptosis repeat; DIABLO, direct IAP-binding protein with low pl; DIAP1, Drosophila inhibitor of apoptosis protein 1; DCP1, Drosophila caspase 1; dOmi, Drosophila Omi; DRICE, Drosophila interleukin-1 $\beta$-converting enzyme; Dronc, Drosophila Nedd2-like caspase; GMR, Glass multimer reporter; GST, glutathione-S-transferase; IAP, inhibitor of apoptosis protein; IBM, IAP-binding motif; IMAGE, Integrated Molecular Analysis of Genomes and their Expression; MTS, mitochondrial targeting sequence; PAGE, polyacrylamide gel electrophoresis; PSORT, protein sorting; RING, really interesting new gene; Smac, second mitochondrial-derived activator; TM, transmembrane; WT, wild type

Received 21.11.07; revised 07.1.08; accepted 07.1.08; Edited by G Melino; published online 15.2.08
} 
Omi/HtrA2 can indirectly induce caspase-dependent apoptosis by degrading and inactivating IAPs. ${ }^{26-28} \mathrm{Omi} / \mathrm{HtrA} 2$ also plays a role in caspase-independent cell death, ${ }^{20}$ perhaps by virtue of being a protease that can cleave vital structural and regulatory components of the cell. In addition to its apoptotic functions, Omi/HtrA2 also plays an important role in cellular homeostasis as a stress sensor and a chaperone. ${ }^{29,30}$

If a cell is not to die, IAP antagonists and IAPs need to be kept apart. In mammals, this is accomplished by the sequestration of the IAP antagonists Smac/DIABLO and Omi/ HtrA2 in the mitochondria. The Drosophila IAP antagonists, however, are located in the cytosol, and translocate to the mitochondria during apoptosis. ${ }^{5}$ Therefore, cell survival is accomplished by either the lack of IAP antagonist gene transcription, or by post-translational modifications of the IAP antagonists, such as phosphorylation or ubiquitination (reviewed in Meier et al.," ${ }^{31}$ Ditzel and Meier, ${ }^{32}$ and Vaux and Silke ${ }^{33}$ ). So far, no similar regulatory mechanisms have been reported for mammalian Smac/DIABLO or Omi/HtrA2.

Since the cell death pathway is well conserved between flies and mammals, we asked whether mitochondria-sequestered IAP antagonists exist in the fly. To address this question, we searched the Drosophila genomic database for homologs of Smac/DIABLO and Omi/HtrA2. In this study, we describe the identification and characterization of a Drosophila mitochondrial serine protease, which we have named dOmi/ HtrA2 (Drosophila Omi) because of its high homology to mammalian Omi/HtrA2. We show that dOmi/HtrA2 is a direct IAP-binding protein and define its role in the regulation of cell death. While this paper was in preparation, Igaki et al. ${ }^{34}$ and Challa et al. ${ }^{35}$ reported the characterization of dOmi/HtrA2 and its interaction with IAPs. Our results extend their observations, by providing the exact $\mathrm{N}$-terminal amino-acid sequences of the mature $\mathrm{dOmi} / \mathrm{HtrA} 2$ isoforms, which differ from those predicted in their studies, and the exact dOmi/ HtrA2 cleavage sites in DIAP1, both determined by direct Edman degradation. Moreover, we show for the first time that thermal stress activates transgenic dOmi/HtrA2 in Drosophila eye discs to induce eye ablation, which could be rescued by transgenic expression of DIAP1. Our biochemical data suggest that DIAP1-mediated polyubiquitination of dOmi/ HtrA2 might be responsible for this protective effect of DIAP1.

\section{Results}

dOmi is localized in the mitochondria and is released into the cytosol during apoptosis. To identify the Drosophila ortholog of mammalian Omi/HtrA2, we searched the $D$. melanogaster public GenBank database using the tBLASTn program for genes that encode proteins with high homology to the human Omi/HtrA2 protein. This resulted in identification of one Drosophila gene (CG8464) on chromosome 3R, which encodes a 422 amino-acid protein (GenBank accession number AAF55062) that has $51 \%$ homology to human Omi, and is designated hereafter as dOmi/HtrA2. Computer analysis with the PSORT (protein sorting) algorithm revealed that dOmi contains a mitochondrial targeting sequence (MTS) at its $\mathrm{N}$ terminus (residues 1-60) followed by a transmembrane domain (TM, residues 64-73), and thus is predicted to be a mitochondrial protein, like its mammalian ortholog. Following the TM domain, dOmi contains a trypsin-like serine protease domain (residues 74-337) and a C-terminal PDZ domain (338-410) (Figure 1a). A full-length cDNA clone (BF499650) was obtained from the Integrated Molecular Analysis of Genomes and their Expression (IMAGE) consortium and was verified by sequencing to encode the full-length dOmi.

To begin to characterize the function of dOmi/HtrA2, we raised a polyclonal antiserum against a bacterially produced dOmi (residues 92-422) and used it to examine the expression and subcellular localization of dOmi. Western blot analysis of whole cell lysate from adult flies revealed the presence of three protein species: the largest species corresponds to the unprocessed dOmi, and two smaller processed species likely represent the mature forms of dOmi (Figure 1b). To determine the subcellular localization of dOmi, we isolated cytosolic and mitochondrial fractions from Sf9 insect cells and subjected them to western blot analysis. The anti-dOmi antibody detected two mature forms of endogenous Omi in the mitochondrial fraction but not in the cytosolic fraction (Figure 1c, first and second lanes). Transient overexpression of full-length dOmi, using a baculovirus encoding full-length dOmi, resulted in a dramatic increase in the expression level of mature dOmi in the mitochondrial fraction of these cells (Figure 1c, fourth lane). However, probably because of baculovirus-induced cell death and overexpression of dOmi, a small amount of mature dOmi was also found in the cytosolic fraction (Figure 1c, third lane). These results indicated that dOmi, like its mammalian ortholog, is a mitochondrial protein.

To determine the effect of an apoptotic stimulus on the subcellular distribution of dOmi, we treated Drosophila S2 cells with UV and then fractionated the cells into cytosolic and mitochondrial fractions. Because dOmi is expressed at low levels in S2 cells, we immunoprecipitated dOmi from the cytosolic and mitochondrial fractions. The immunoprecipitates were then fractionated by SDS-polyacrylamide gel electrophoresis (PAGE) and analyzed by immunoblotting. As shown in Figure 1d, UV treatment of $S 2$ cells resulted in translocation of dOmi from mitochondria to the cytosol. These results indicate that $\mathrm{dOmi}$, like its mammalian ortholog, is released from the mitochondria during apoptosis, and they further suggest that its function might parallel that of mammalian Omi.

To verify that dOmi possesses proteolytic activity, we incubated purified bacterially-expressed recombinant dOmi with ${ }^{35}$ S-labeled $\beta$-casein. As shown in Figure 1e, the recombinant dOmi was able to cleave $\beta$-casein into smaller fragments visible by autoradiography. We also immunoprecipitated endogenous dOmi from both uninfected and dOmibaculovirus-infected Sf 9 cells with the dOmi-specific antibody and assayed the proteolytic activity of the immunoprecipitates with ${ }^{35}$ S-labeled $\beta$-casein (Figure 1e). This experiment revealed that both endogenous Sf9-Omi and recombinant dOmi could cleave ${ }^{35} \mathrm{~S}$-labeled $\beta$-casein, indicating that insect Omi is proteolytically active.

Maturation of dOmi in the mitochondria generates two isoforms with distinct IBMs. Maturation of Omi/HtrA2 in 
a
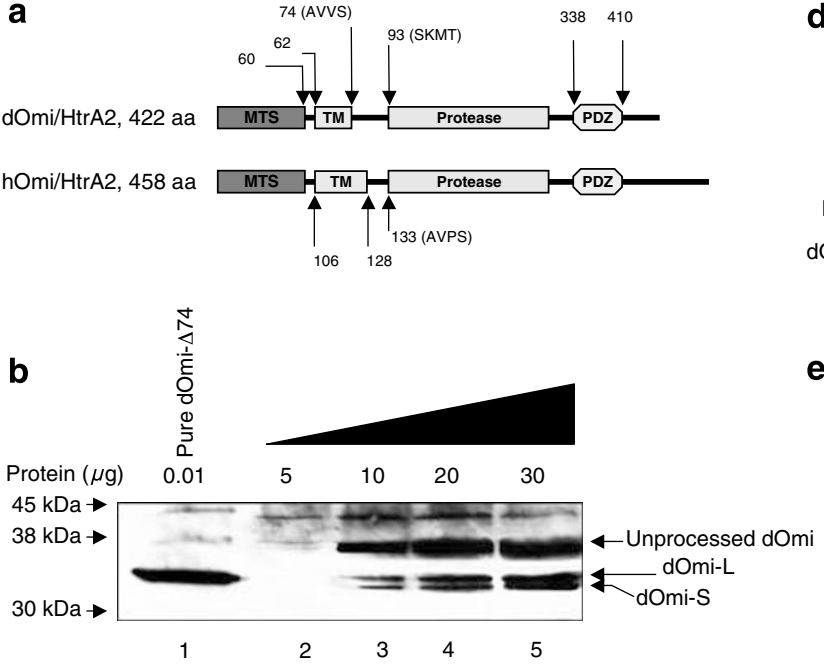

C

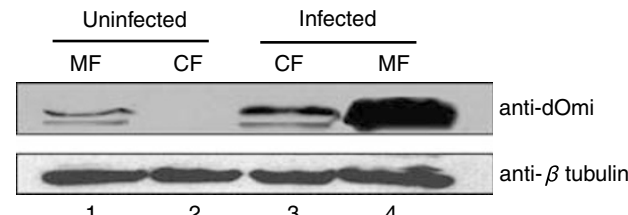

d

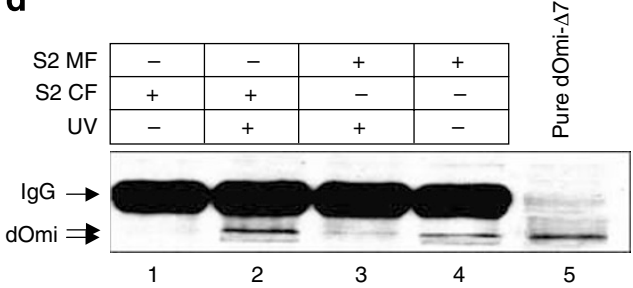

e

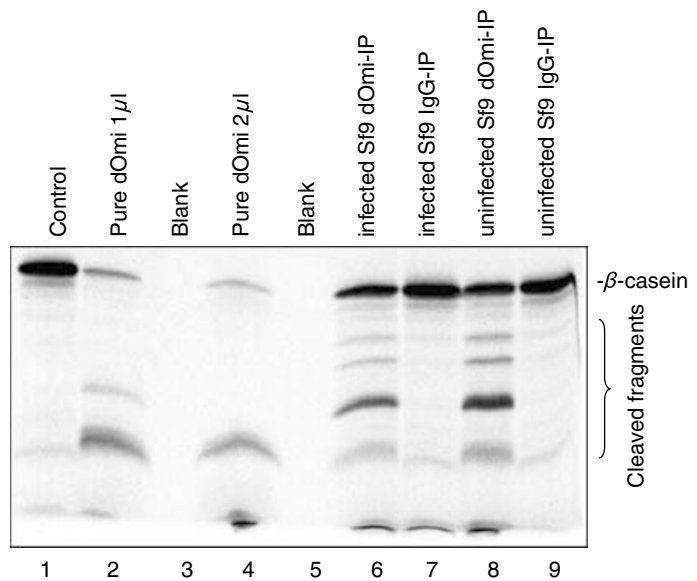

Figure 1 Expression, subcellular localization, and proteolytic activity of dOmi. (a) Schematic representation of the domain structure of Drosophila Omi in comparison to human Omi/HtrA2 showing the MTS, TM domain, protease domain, and the C-terminal PDZ regulatory domain. The two processing sites at A74 and A93 that give rise to the mature dOmi-L and dOmi-S, respectively, are indicated. Mature human Omi/HtrA2 is generated by processing at A133. (b). Endogenous expression of dOmi in whole fly lysates. Increasing amounts of fly lysate (5-30 $\mu \mathrm{g}$ ) were fractionated by SDS-PAGE and then western blotted with an anti-dOmi antibody. (c) Western blot analysis of mitochondrial (MF) and cytosolic (CF) fractions of uninfected Sf9 cells (lanes 1 and 2) and dOmi-baculovirus-infected Sf9 cells (lanes 3 and 4) with anti-dOmi polyclonal antibody. Notice that endogenous Sf9-Omi is detected in the MF, but not in the CF of uninfected Sf9 cells (lane 1). Overexpressed dOmi is predominantly present in the mitochondrial fraction (lane 4). A low level of dOmi is detectable in the cytosolic fraction due to overexpression (lane 3). (d) During UV-induced cell death, endogenous dOmi translocates from the mitochondria to the cytosol, in Drosophila S2 cells. After UV treatment, cytosolic (CF) and mitochondrial (MF) fractions were immunoprecipitated with anti-dOmi antibody and then analyzed by western blotting with the same antibody. (e) Proteolytic activity of insect Omi toward the non-physiological substrate $\beta$-casein. Endogenous Sf9-Omi (lanes 8 and 9) or overexpressed dOmi (lanes 6 and 7) was immunoprecipitated (IP) from uninfected Sf9 or dOmi-baculovirus-infected Sf9 cells, respectively, with dOmi antibody or nonspecific pre-immune serum (IgG) as indicated. The IPs were incubated with ${ }^{35} \mathrm{~S}$-labeled $\beta$-casein and then fractionated by SDS-PAGE followed by autoradiography. Lane 1 shows $\beta$-casein after incubation with a buffer control. Lanes 2 and 4 show $\beta$-casein after incubation with pure recombinant dOmi

the mitochondria involves proteolytic removal of the $\mathrm{N}$-terminal MTS. In the case of human Omi/HtrA2, this maturation exposes a conserved $\mathrm{N}$-terminal IBM with the sequence AVPS, ${ }^{20-23}$ where $\mathrm{Ala}$ is the first $\mathrm{N}$-terminal residue. After the release of mature $\mathrm{Omi} / \mathrm{HtrA} 2$ into the cytosol during cell death, it binds to IAPs via its IBM and prevents them from binding to active caspases and/or directly degrades them into inactive fragments. ${ }^{26-28}$ To determine if dOmi maturation in the mitochondria generates an N-terminal IBM, we expressed the full-length precursor form of dOmi with a C-terminal $\mathrm{His}_{6}$-tag in $\mathrm{Sf9}$ insect cells using a recombinant baculovirus. This recombinant $\mathrm{dOmi}$ was affinity purified on Talon beads and fractionated by SDS-PAGE. Coomassie blue staining (Figure 2a) revealed two processed isoforms similar in size to the mature isoforms of dOmi observed in fly lysates by western blotting (Figure 1b), in addition to the unprocessed precursor form. Sequence analysis of the two processed isoforms by automated Edman degradation revealed that the larger isoform (dOmi-L) begins with Ala75, whereas the smaller isoform (dOmi-S) begins with Ser94. The N-terminal sequence of the first four residues of dOmi-L (AVVS) aligns very well with the canonical IBM found in other IAP-binding proteins
(Figure 2b). In contrast, the N-terminal sequence of the first four residues of the smaller dOmi-S isoform (SKMT) does not align with the canonical IBM. Nevertheless, other proteins with IBMs that begin with Ser residues have been shown to bind to IAPs, such as the small subunit of caspase-7 $(\mathrm{SGPI})^{36}$ and glutamate dehydrogenase (SEAV). ${ }^{37}$ These observations suggested that the two mature dOmi isoforms might bind to Drosophila IAPs, just as mature Omi/HtrA2 binds to mammalian IAPs.

To test whether the mature $\mathrm{dOmi}$ isoforms could bind to IAPs, we immobilized C-terminal $\mathrm{His}_{6}$-tagged dOmi-L and dOmi-S isoforms on Talon beads and then incubated the beads at $4{ }^{\circ} \mathrm{C}$ with in vitro-translated ${ }^{35} \mathrm{~S}$-labeled XIAP and DIAP1. Talon bead-bound glutathione-S-transferase (GST)$\mathrm{His}_{6}$ was used as a negative control. Figure 2c shows that both dOmi-L and dOmi-S bind strongly to both XIAP and DIAP1. The binding efficiency of the mature dOmi isoforms to XIAP and DIAP1 was comparable to that of mature forms of human Omi/HtrA2 and Smac/DIABLO. However, when the first alanine residue of the IBM was substituted with proline (fourth lane upper panel and fifth lane lower panel) the binding of dOmi-L to both XIAP and DIAP1 was significantly reduced, confirming the role of the first Ala in IAP binding. We did not 
a

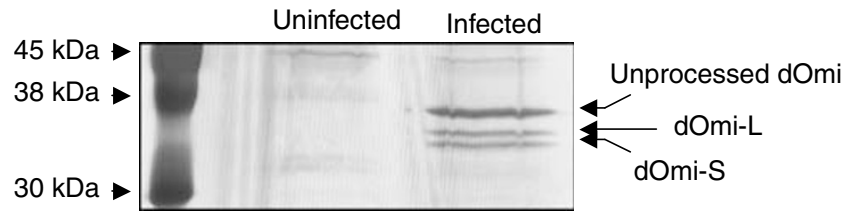

b

\begin{tabular}{|c|c|c|c|c|c|c|c|}
\hline Reaper & A V & $J \mathrm{~A}$ & $\mathrm{~F}$ & $\mathrm{Y}$ & I & D & \\
\hline Grim & A I & {$[\mathrm{A}$} & $Y$ & $\mathrm{~F}$ & I & $\mathrm{P}$ & D \\
\hline Sickle & A I & I $\mathrm{P}$ & $\mathrm{F}$ & $\mathrm{F}$ & $\mathrm{E}$ & $\mathrm{E}$ & $\mathrm{E}$ \\
\hline Hid & A V & $J \mathrm{P}$ & $\mathrm{F}$ & $\mathrm{Y}$ & L & $\mathrm{P}$ & E \\
\hline Jafrac-2 & A $K$ & K $P$ & $\mathrm{E}$ & $\mathrm{D}$ & $\mathrm{N}$ & $E$ & $S$ \\
\hline Smac/DIABLO & A V & T $\mathrm{P}$ & I & A & $Q$ & $\mathrm{~K}$ & $S$ \\
\hline Omi/HtrA2 & A $V$ & I $\mathrm{P}$ & S & $\mathrm{P}$ & $P$ & $\mathrm{P}$ & A \\
\hline Caspase 9 & A $\mathrm{T}$ & $\Gamma \mathrm{P}$ & $\mathrm{F}$ & Q & $\mathrm{E}$ & 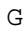 & 1 \\
\hline $\mathrm{GdH}$ & $S E$ & $\mathrm{~A}$ & V & A & $D$ & $\mathrm{R}$ & E \\
\hline Capase 7 & $S$ G & $\mathrm{P}$ & I & $\mathrm{N}$ & D & $\mathrm{T}$ & I \\
\hline domi-I & A V & $J \mathrm{~V}$ & $S$ & A & A & A & I \\
\hline domi-S & $\mathrm{S} \mathrm{K}$ & $\mathrm{K} M$ & $\mathrm{~T}$ & G & $\mathrm{R}$ & $\mathrm{R}$ & $\mathrm{F}$ \\
\hline
\end{tabular}

c

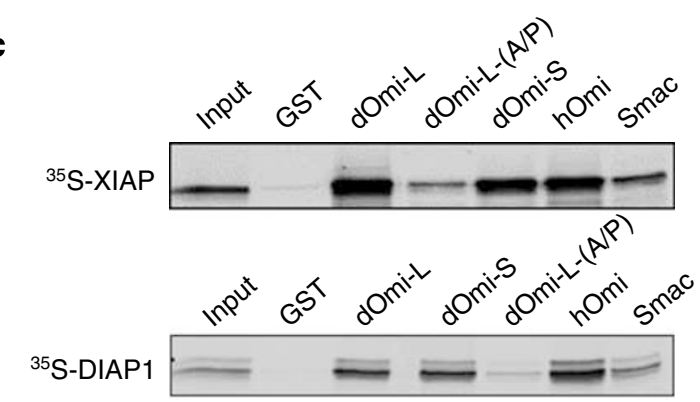

Figure 2 The $\mathrm{N}$ terminus of mature dOmi harbors an IAP-binding motif. (a) Coomassie blue-stained membrane showing full-length (unprocessed) dOmi and the two processed mature forms dOmi-L and dOmi-S. This preparation of dOmi was purified from the mitochondrial fraction of dOmi-baculovirus-infected Sf9 cells on Talon beads and then fractionated by SDS-PAGE. (b) Chart showing the sequences of processed $\mathrm{N}$ termini of several IBM-containing proteins. The conserved IBM sequence is shown in red. dOmi-L and dOmi-S share similarity with other IBMs. (c) In vitro interaction with ${ }^{35} \mathrm{~S}$-labeled XIAP (upper panel) or DIAP1 (lower panel) of $\mathrm{His}_{6}$-tagged variants of dOmi (dOmi-L, dOmi-A/P, and dOmi-S) immobilized on Talon resin. His $_{6}$-tagged hOmi (lane 6) and Smac (lane 7) were used as positive controls. His $s_{6}$-tagged GST (lane 2) was used as a negative control. dOmi-L-(A/P) represents a mutant dOmi-L in which the first Ala residue in the IBM is mutated to Pro

observe any interaction between GST-His ${ }_{6}$ and DIAP1 or $\mathrm{XIAP}$, indicating that the interaction between dOmi isoforms and IAPs is specific.

dOmi binds to and cleaves Drosophila IAPs. IAP degradation is an important mechanism for caspase activation during development. ${ }^{33}$ The Drosophila IAP antagonists, which are induced during development or DNA damage, bind to the BIR2 (baculovirus inhibitor of apoptosis repeat 2) domain of DIAP1, resulting in its ubiquitination and subsequent degradation through the proteasomal pathway. ${ }^{38}$ Given that dOmi is a serine protease, we asked whether binding of mature dOmi isoforms to Drosophila IAPs could also lead to their degradation by the proteolytic activity of dOmi. Therefore, we incubated DIAP1 or DIAP2 with increasing amounts of wild-type (WT) dOmi-L, or an inactive S266A-dOmi-L mutant, at room temperature for 1h. As shown in Figure 3a, WT dOmi-L, but not the proteolytically inactive S266A-dOmi-L mutant, cleaved both DIAP1 and DIAP2 into smaller fragments in a dosedependent manner, indicating that these proteins are physiological substrates for dOmi.

To determine whether the dOmi-generated DIAP1 or DIAP2 fragments contain intact BIR domains and therefore would remain associated with dOmi, we incubated DIAP1 or DIAP2 with Talon bead-bound WT dOmi-L, or the inactive S266A$\mathrm{dOmi}-\mathrm{L}$ mutant, at room temperature for $1 \mathrm{~h}$. Talon beadbound GRIM and GST-His ${ }_{6}$ were used as controls. After the incubation period, the beads were washed, and the beadbound complexes were fractionated by SDS-PAGE and visualized by autoradiography. As shown in Figure 3b (upper left panel), cleavage of DIAP1 by dOmi-L produced a distinct cleavage fragment that remained associated with the beadbound dOmi-L, indicating that it contains an intact BIR domain. In contrast, none of the DIAP2 fragments remained associated with dOmi-L, indicating that cleavage of DIAP2 by dOmi-L destroys the BIR domains of DIAP2 (Figure $3 b$, lower left panel). As expected no cleavage of DIAP1 or DIAP2 was observed when they were bound to the proteolytically inactive S266A-dOmi-L mutant (right panels). In addition, no degradation of the GRIM-bound DIAP1 or DIAP2 was observed and no significant binding of DIAPs to GST-His 6 was detected.

Cleavage of DIAP1 by dOmi generates a distinct cleavage product that retains the ability to interact with dOmi (Figure $3 b$ ). To identify this cleavage site, we produced in bacteria a recombinant DIAP1 fusion protein with a GST tag at its $\mathrm{C}$ terminus. The DIAP1-C-GST fusion protein was immobilized on glutathione agarose beads and then bound to bacterially produced mature dOmi-L or dOmi-S at $4^{\circ} \mathrm{C}$ for $1 \mathrm{~h}$. The bead-bound complexes were washed and then analyzed by SDS-PAGE. As shown in Figure 3c, binding of either the dOmi-L or dOmi-S to DIAP1-C-GST produced a 60kDa DIAP1 cleavage product. The cleavage did not dissociate the DIAP1-dOmi complex from the glutathione sepharose beads, indicating that the cleavage site is located in the $\mathrm{N}$ terminus of DIAP1, since the GST tag is present at the $C$ terminus of DIAP1. Indeed, N-terminal sequence analysis of the cleavage product by automated Edman degradation revealed that cleavage occurred in the scissile peptide bond between I165 and G166 of DIAP1, which is located in the linker region between the BIR1 and BIR2 domains.

The selectivity for a nonpolar aliphatic residue at position $\mathrm{P} 1$ (immediately $\mathrm{N}$-terminal to the scissile bond) appears to be similar to that of human Omi/HtrA2. ${ }^{39}$ To determine the effect of substitution of I165 in the cleavage site with a charged Asp residue on dOmi-mediated cleavage of DIAP1, we incubated either WT or mutant I165D DIAP1-C-GST with dOmi-L and then analyzed the cleavage products by SDS-PAGE. As shown in Figure 3d, substitution of the 1165 with Asp reduced cleavage of DIAP1 by dOmi-L and dOmi-S but did not completely abolish it. Interestingly, the 1165D substitution shifted cleavage to an adjacent site, as evidenced by the appearance of two cleavage products in the mutant I165D DIAP1-C-GST instead of the one cleavage product seen with the WT protein. Since there is an lle at position 162, the additional cleavage product seen with the 1165D mutant could be the result of cleavage at the scissile bond between Ile162 
a

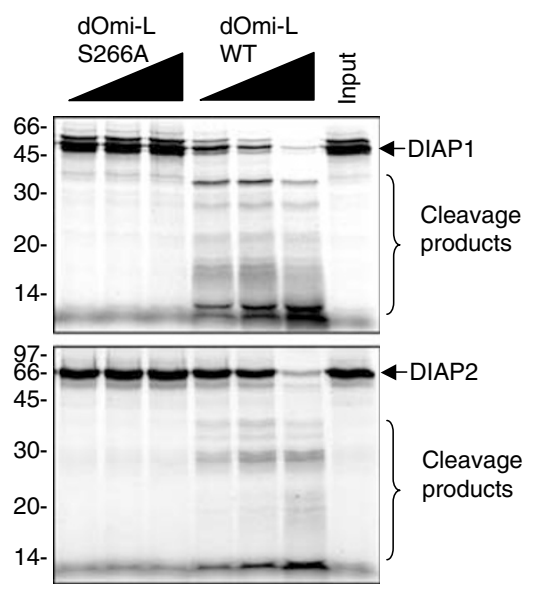

C

\begin{tabular}{r|c|c|c|c|}
\cline { 2 - 5 } dOmi-S-WT & - & + & - & - \\
\cline { 2 - 5 } dOmi-L-WT & - & - & - & + \\
\cline { 2 - 5 } DIAP1-C-GST & - & + & + & + \\
\cline { 2 - 5 } GST & + & - & - & - \\
\hline
\end{tabular}

b

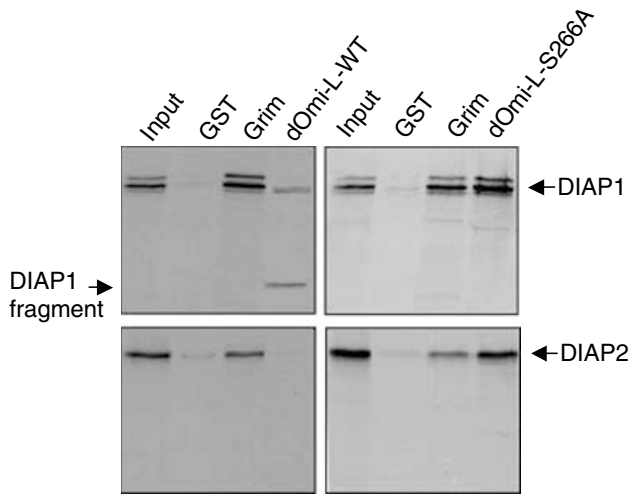

d

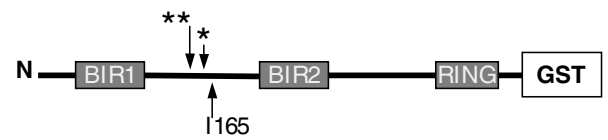

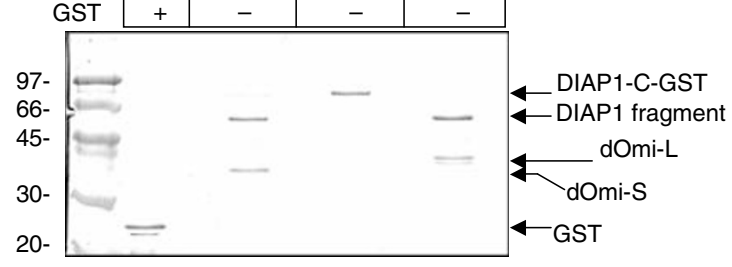

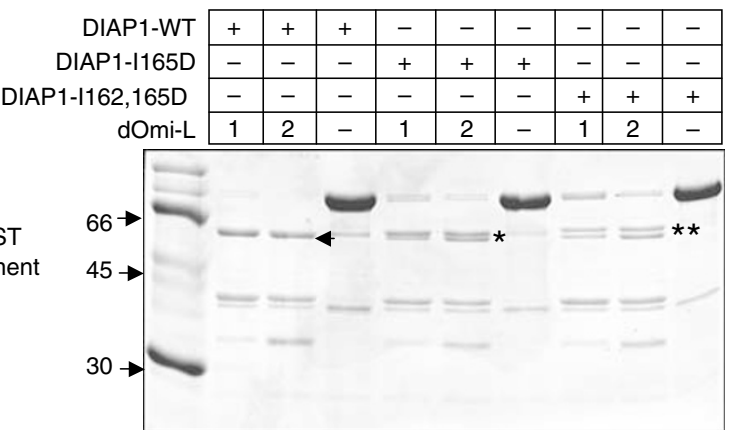

Figure 3 dOmi binds and degrades DIAP1 and DIAP2. (a) ${ }^{35}$ S-labeled DIAP1 (upper panel) or DIAP2 (lower panel) were incubated with increasing amounts (50, 100, and $250 \mathrm{nM}$ ) of wild-type dOmi or the inactive S266A mutant dOmi for $1 \mathrm{~h}$ at $25^{\circ} \mathrm{C}$ as indicated. Samples were then fractionated by SDS-PAGE followed by autoradiography. (b)

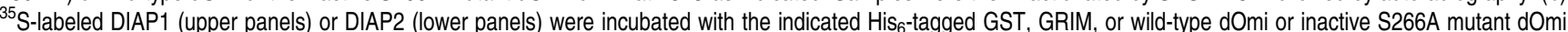
immobilized on Talon beads, for $1 \mathrm{~h}$ at $4^{\circ} \mathrm{C}$. After binding, the bead-bound complexes were washed and then incubated at room temperature for an additional $30 \mathrm{~min}$. Samples were then fractionated by SDS-PAGE followed by autoradiography. (c) Coomassie-stained SDS gel showing cleavage of DIAP1 by dOmi-L or dOmi-S. dOmi-L or dOmi-S was bound to C-terminal GST-tagged DIAP1 (DIAP1-C-GST) or GST on glutathione sepharose beads for $1 \mathrm{~h}$ at $4^{\circ} \mathrm{C}$. After binding, the bead-bound complexes were washed and then incubated at room temperature for an additional $30 \mathrm{~min}$. Samples were then fractionated by SDS-PAGE followed by Coomassie staining. (d) Effects of DIAP1 linker mutations on degradation of DIAP1 by mature dOmi-L. Equal amounts of bacterially expressed full-length wild-type (WT) DIAP1 (lanes 2-4), DIAP1-I165D (lanes 5-7), or DIAP1-I162 and 165D (lanes 8-10) were bound to Glutathione Sepharose ${ }^{\mathrm{TM}} 4 \mathrm{~B}$ beads and then incubated with 1 or $2 \mu$ of mature dOmi-L for $1 \mathrm{~h}$ at $22^{\circ} \mathrm{C}$ as indicated. After the incubation period, the bead-bound proteins were washed and then subjected to SDS-PAGE followed by Coomassie staining. Notice that DIAP1 mutants (lanes 5, 6, 8, and 9) undergo incomplete degradation, whereas the WT DIAP1 (lanes 2 and 3) undergoes complete degradation. There is also a distinct pattern of cleavage of both DIAP-1/165D (denoted by *) and DIAP1-I162 and 165D (denoted by **), indicating a shift in the cleavage site when compared with the WT DIAP1 (denoted by $\leftarrow$ ). The cleavage sites in the WT and mutant DIAP1-GST are illustrated schematically above the gel. Arrows indicate the cleavage sites

and Met163. However, substitution of I165 and I162 with Asp residues reduced but did not abolish cleavage, and from the size of the new products it appears that the cleavage has shifted to a scissile bond immediately $\mathrm{N}$-terminal to lle165 and lle162 (Figure 3d). Altogether, these results indicate that binding of dOmi to DIAP1 results in selective cleavage by dOmi of the linker region between BIR1 and BIR2 of DIAP1.

Since dOmi was still bound to the $60-\mathrm{kDa}$ bead-bound DIAP1 cleavage product (Figure $3 b-d$ ), this indicates that dOmi binds preferentially to the BIR2 domain of DIAP1. To confirm this, we produced N-terminal GST-tagged BIR1 (GST-BIR1-1-165) and BIR2 (GST-BIR2-RING-166-438) fusion proteins in bacteria. These proteins were incubated with proteolytically inactive S266A dOmi-L or dOmi-S mutants and the resulting protein complexes were then bound to glutathione sepharose beads. Analysis of the bead-bound complexes by SDS-PAGE revealed that dOmi isoforms bound to both the GST-BIR2-RING-166-438 and GST-BIR1-1-165 (Figure 4a). However, the affinities of the dOmi isoforms were higher toward GST-BIR2-RING-166-438 than toward GST-BIR1-1-165 (Figure 4a), suggesting that dOmi isoforms preferentially associate with the BIR2 domain of DIAP1. Interestingly, when the GST-BIR1-1-165 or GST-BIR2RING-166-438 was bound to WT dOmi-L or dOmi-S, degradation of GST-BIR1-1-165, but not GST-BIR2-RING166-438, was observed (Figure 4b). These results indicate that dOmi isoforms bind preferentially to the BIR2 domain of DIAP1 but preferentially cleave the linker region and the BIR1 domain (Figure 4c).

To provide additional evidence that binding of dOmi to the BIR1-linker region of DIAP1 results in additional cleavages $\mathrm{N}$-terminal to 1165 , we incubated purified dOmi-L or dOmi-S with ${ }^{35}$ S-labeled DIAPI-BIR1 (residues 1-165). As shown in Figure $4 \mathrm{~d}$, incubation of dOmi-L or dOmi-S with the 
a GST-BIR2-RING-166-438 GST-BIR1-1-165 dOmi-S (S266A) GST \begin{tabular}{c|c|c|}
\hline- & + & \\
\hline- & - & + \\
\hline+ & + & + \\
\hline- & - & \\
\hline- & - & - \\
\hline- & - &
\end{tabular} 66-

b

\begin{tabular}{r|c|c|c|c|c|c|}
\cline { 2 - 7 } dOmi-L-wt & - & + & - & - & + & - \\
\cline { 2 - 7 } dOmi-S-wt & - & - & + & - & - & + \\
\cline { 2 - 7 } & - & - & - & + & + & + \\
\cline { 2 - 7 } & + & + & + & - & - & -
\end{tabular}

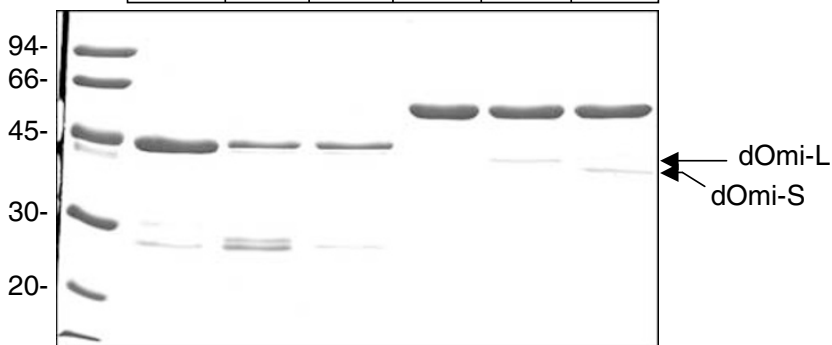

C
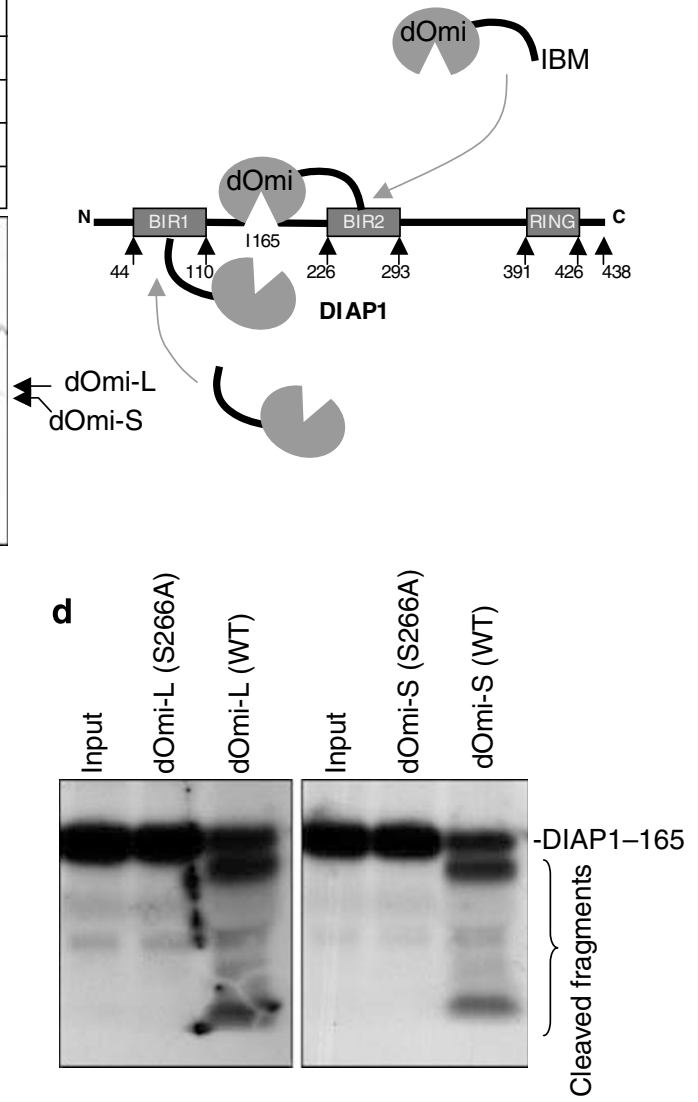

Figure 4 dOmi binds preferentially to the BIR2 domain of DIAP1. (a) Interaction of full-length DIAP1 and the isolated DIAP1-BIR domains with mature protease-inactive dOmi-L or dOmi-S. S266A dOmi-L or dOmi-S was bound to C-terminal GST-tagged DIAP1 (DIAP1-C-GST), or N-terminal GST-tagged BIR1 (GST-BIR1-1-165) or BIR2-RING (GST-BIR2-RING-166-438), or GST on Glutathione Sepharose ${ }^{\mathrm{TM}} 4 \mathrm{~B}$ beads at $4^{\circ} \mathrm{C}$ for $1 \mathrm{~h}$ as indicated. After binding, the bead-bound complexes were washed and then fractionated by SDS-PAGE followed by Coomassie staining. Notice that both mature forms of dOmi (dOmi-L and dOmi-S) bind to full-length DIAP1-C-GST and preferentially to GST-BIR2-RING-166-438. (b) Same as in (a) but using wild-type (WT) dOmi-L and dOmi-S. Notice that both mature forms of WT dOmi (dOmi-L and dOmi-S) cleave GSTBIR1-1-165, but not GST-BIR2-RING-166-438. (c) Domain map of DIAP1 showing BIR1, BIR2, and RING finger domains, along with the dOmi cleavage site in the linker region between BIR1 and BIR2 as determined by automated Edman degradation. The diagram illustrates dOmi binding to the BIR1 and BIR2 domains of DIAP1 via its IBM, and cleaving the linker region between BIR1 and BIR2 of DIAP1. (d) ${ }^{35}$ S-labeled DIAP1-BIR1-linker domain (DIAP1-165) was incubated with WT dOmi (WT) or inactive S266A mutant dOmi (S266A) for $1 \mathrm{~h}$ at room temperature as indicated. Samples were then fractionated by SDS-PAGE followed by autoradiography

DIAP1-BIR1-linker region resulted in its further degradation into smaller fragments, suggesting that dOmi not only cleaves at 1165 but also further degrades the bound BIR1-linker region to completely inactivate it. These results emphasize the notion that the proteolytic activity of Omi is not promiscuous, but selectively targets specific regions in protein substrates, such as the BIR1 and linker region of DIAP1.

dOmi relieves caspases from DIAP1 inhibition. DIAP1 has been shown to inhibit Drosophila effector caspases DCP-1 and DRICE (Drosophila interleukin-1 $\beta$-converting enzyme) via its BIR1 domain. ${ }^{10}$ The ability of dOmi to bind to DIAP1 and degrade its BIR1-linker region suggests that dOmi might abolish DIAP1's caspase-inhibitory activity. Therefore, we tested the effects of purified dOmi-L and dOmi-S proteins on the ability of DIAP1 to inhibit DCP-1 and DRICE using an in vitro caspase activation system. Incubation of DCP-1 or DRICE with DIAP1 resulted in inhibition of their caspase activity, as determined with the tetrapeptide substrate DEVD-AFC. However, addition of dOmi-L or dOmi-S to the reaction mixture resulted in almost complete inhibition of DIAP1 activity, as evidenced by the restoration of Drosophila caspase 1 (DCP1) and DRICE activities (Figure $5 a$ and $b$ ). Point mutations in the IBM of dOmi-L (A75P) or dOmi-S (S94P) completely abolished their caspase promoting activity, suggesting that the ability of dOmi-L and dOmi-S to inhibit DIAP1 activity is dependent predominantly on their ability to bind to DIAP1. Interestingly, point mutations in the protease active site (S266A) significantly reduced, but did not abolish, the caspase-promoting activity of dOmi-L and dOmi-S, indicating that degradation of DIAP1 by dOmi is also important for maximum caspase activation. Collectively, our results indicate that dOmi specifically targets the BIR1 domain of DIAP1, and that both its IBM and protease activity are required for maximum antagonism of the caspase-inhibitory activity of DIAP1. 

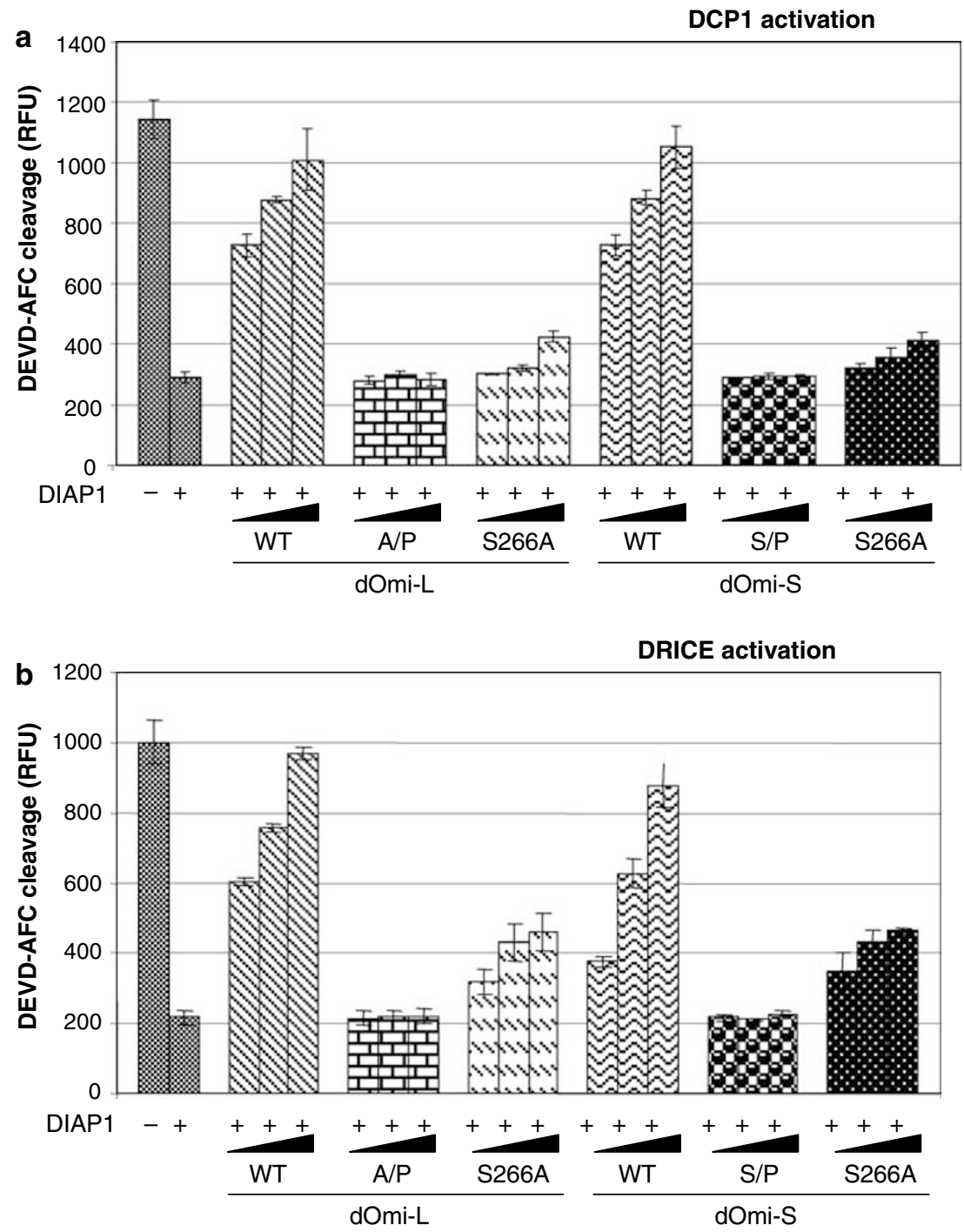

Figure 5 dOmi inactivates the caspase-inhibitory activity of DIAP1. (a) Inhibition of DIAP1 activity by wild-type dOmi-L and dOmi-S. Purified DCP1 (10 nM) was preincubated with DIAP1 $(250 \mathrm{nM})$, and then increasing amounts $(50,100$, and $250 \mathrm{nM})$ of the indicated dOmi variants were added. The reactions were carried out in the presence of DEVD-AFC $(100 \mu \mathrm{M})$ as a substrate for $15 \mathrm{~min}$ at $25^{\circ} \mathrm{C}$. The activity of each reaction is represented as relative fluorescence units (RFUs) released from the substrate. dOmi$\mathrm{L}-(\mathrm{A} / \mathrm{P})$ represents a mutant dOmi-L in which the first Ala residue in the IBM is mutated to Pro. dOmi-S-(S/P) represents a mutant dOmi-S in which the first Ser residue in the IBM is mutated to Pro. (b) Same as (a) except that DCP1 was replaced with purified DRICE (10 nM) and pre-incubated with $200 \mathrm{nM}$ DIAP1

DIAP1 inhibits dOmi-induced cell death in Drosophila eye discs. Omi is sequestered in the mitochondria and is kept in an inactive conformation through extensive intramolecular interactions between its PDZ and protease domains. ${ }^{40}$ Recent studies suggest that under stress conditions, Omi is activated either by binding of its PDZ domain to misfolded proteins or by p38-mediated phosphorylation to protect the mitochondria. ${ }^{41,42}$ To examine the effect of dysregulation of the proteolytic activity of mitochondrially localized Omi on Drosophila eye development, we generated transgenic flies expressing either the full-length dOmi, or a constitutively active dOmi (dOmi- $\triangle \mathrm{PDZ}$ ), under the control of the Glass transcriptional activator, using the pGMR (Glass multimer reporter) vector. Glass is expressed during eye development, starting in the larval eye imaginal discs (the precursors of adult Drosophila eyes). It continues to be expressed specifically in the eye during pupal and adult development. ${ }^{43}$ We found that in most transgenic lines (14/19 lines), even in homozygotes, pGMR-derived expression of fulllength mitochondrial-localized dOmi did not disrupt eye development (Figure 6a, panel 2). In contrast, pGMRderived expression of the constitutively active dOmi- $\triangle \mathrm{PDZ}$ caused eye ablation in most lines (25/31 lines) (Figure 6a, panel 3). These results show that overexpression of WT $\mathrm{dOmi}$ in its proper environment (mitochondria) does not lead to cell death, presumably because dOmi, like mammalian Omi, is kept in an inactive conformation under normal conditions. ${ }^{41,42}$ This is consistent with the survival function of Omi in the mitochondria. ${ }^{29,30}$ However, dysregulation of the protease activity of dOmi by deleting the PDZ domain causes cell death, suggesting that the activity of dOmi must be tightly regulated to allow cell survival. 


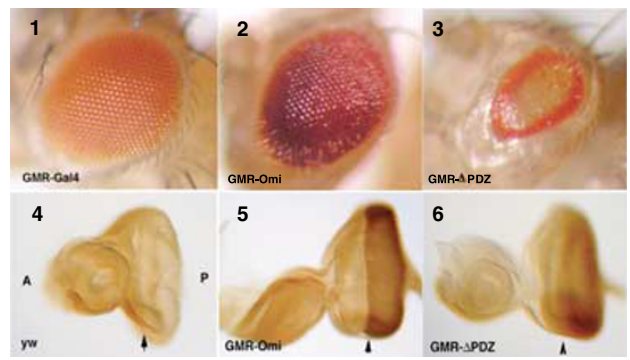

b

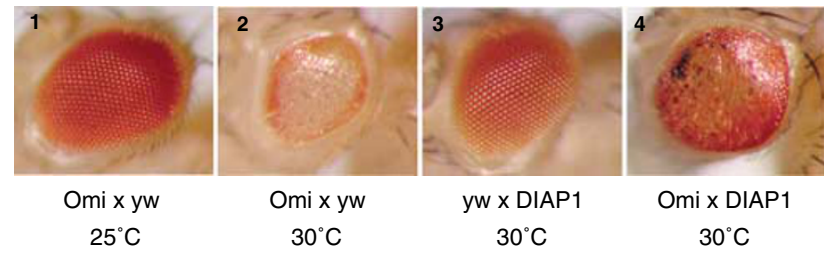

c d

Extract - - + + + $\operatorname{DIAP1}(\mu \mathrm{l})-2-12$

$\mathrm{Ub}+++++$

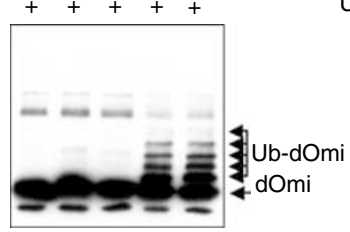

dOmi-A74P

$-++$

Omi-WT

$++$

12

$\mathrm{Ub}++++++$
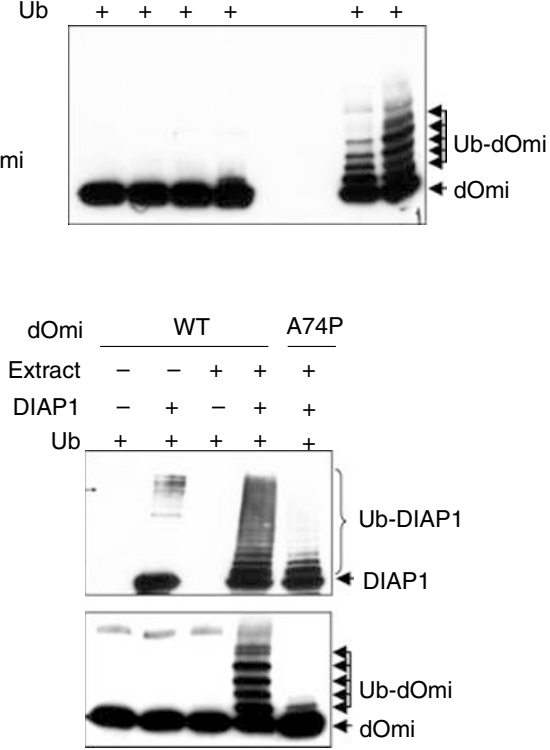

Figure 6 Effects of dOmi overexpression on Drosophila eye development. (a) Effect of ectopically expressed full-length dOmi and $\triangle \mathrm{PDZ}-\mathrm{dOmi}$ on Drosophila eye development. Panel 1 shows a wild-type (WT) eye (GMR-Gal4 as a negative control); panel 2, typical eye phenotype with GMR-driven overexpression of full-length dOmi (homozygous transgene); panel 3 , eye phenotype caused by GMR-driven overexpression of dOmi in which a negative regulatory domain has been removed ( $\triangle \mathrm{PDZ}$ homozygous transgene). Panels 4-6 show Drosophila eye imaginal discs immunostained with anti-dOmi. Note the increased level of dOmi in panels 5 and 6 relative to the wild type (panel 4), and that dOmi is overexpressed at a similar level as the $\triangle \mathrm{PDZ}$ form. (b) Activation of dOmi by thermal stress induces eye ablation, and expression of DIAP1 suppresses this eye phenotype. Transgenic Drosophila (females, transgene on the $\mathrm{X}$ chromosome) expressing full-length dOmi in the eye showed a normal eye phenotype when maintained at $25^{\circ} \mathrm{C}$ (first panel). However, when kept at $30^{\circ} \mathrm{C}$ during larval stages (see Materials and Methods), they showed a severe eye ablation phenotype (second panel). This ablation was entirely dependent on expression of dOmi (third panel: control showing expression of DIAP1 alone; WT flies kept at $30^{\circ}$ as well as DIAP1overexpressing flies kept at $25^{\circ}$ also showed a WT phenotype, not shown). This eye phenotype was suppressed by co-expressing DIAP1 in the eye (fourth panel). (c) DIAP1 induces ubiquitination of dOmi. Purified $\mathrm{His}_{6}$-tagged dOmi (100 ng) was incubated with ubiquitin ( $\left.3 \mu \mathrm{g}\right)$, DIAP1 (400 or $\left.800 \mathrm{ng}\right)$ and Drosophila embryonic extracts $(10 \mu \mathrm{g})$ in a ubiquitination buffer $(20 \mu \mathrm{l})$ for $1 \mathrm{~h}$ at $37^{\circ} \mathrm{C}$ as indicated. The reactions were then analyzed by western blotting with anti-His antibody to detect dOmi ubiquitination. Notice polyubiquitination of dOmi in the reactions that contain DIAP1 and Drosophila embryonic extracts (fourth and fifth lanes), but not in the absence of DIAP1 (third lane). (d) DIAP1-induced ubiquitination of dOmi is dependent on an intact IBM motif in dOmi. Purified His 6 -tagged mutant dOmi-A74P (first to fourth lanes) or dOmi-WT (fifth and sixth lanes) was subjected to ubiquitination and then analyzed as in (c). Note that DIAP1 does not induce ubiquitination of the dOmi-A74P mutant, because the A74P mutation destroys the N-terminal IAP-binding motif of dOmi. (e) dOmi induces auto-ubiquitination of DIAP1. Purified His 6 -tagged dOmi-WT (first through fourth lanes) or mutant dOmiA74P (fifth lane) was incubated with or without DIAP1 as indicated in a ubiquitination reaction similar to that described in (c). The reaction products were then analyzed by western blotting with anti-DIAP1 antibody (upper panel), or anti-His antibody (lower panel)

Recent results suggest that Omi is activated by stress stimuli via the 338 stress kinase pathway. ${ }^{41}$ Since thermal stress is known to activate the p38 pathway, we examined the effect of thermal stress on eye development of the WT dOmioverexpressing flies. GMR-dOmi larvae were either kept at room temperature or stressed by incubation at $30^{\circ} \mathrm{C}$ for 5 days. The eyes of the newly enclosed adult flies were then examined. As shown in Figure $6 \mathrm{~b}$ (panel 1), the eyes of GMROmi flies that were kept at room temperature were normal. In contrast, the eyes of the heat-stressed GMR-dOmi flies showed abnormalities similar to those observed in the GMRdOmi- $\Delta$ PDZ flies (Figure $6 \mathrm{~b}$, panel 2), indicating that dOmi activity is increased under stress conditions. This phenotype was not observed in $30^{\circ} \mathrm{C}$-treated WT flies that do not overexpress dOmi, indicating that this effect is mediated by increased dOmi activity under thermal stress (Figure 6b, panel 3).

Considering that DIAP1 can specifically interact with dOmi, we tested the possibility that DIAP1 might function to protect cells from increased dOmi activity under stress conditions.
Therefore, we crossed the GMR-dOmi flies with GMR-DIAP1 flies, and tested the effect of thermal stress on eye development. Interestingly, overexpression of DIAP1 greatly reduced the thermal stress-induced eye abnormalities induced by overexpressed dOmi (Figure 6b, panel 4; compare to panel 2).

DIAP1 polyubiquitinates dOmi. Given that DIAP1 possesses a ubiquitin ligase activity, it is possible that DIAP1 antagonizes the activity of dOmi by directly targeting it for ubiquitination and proteasomal degradation. To examine this possibility, we incubated dOmi-L with or without DIAP1 in a reaction mixture containing Drosophila embryonic extracts and ubiquitin. As shown in Figure 6c, dOmi was polyubiquitinated in the presence of DIAP1, but not in its absence. Moreover, DIAP1-induced ubiquitination of dOmi was dependent on an intact IBM motif of dOmi, as substitution of Ala75 with Pro completely prevented ubiquitination of dOmi (Figure 6d). Since the A75P mutant dOmi-L has a very low affinity toward DIAP1, compared with 
WT dOmi-L (Figure 2c), these results indicate that a strong interaction of dOmi with DIAP1 is required to stimulate the E3 ligase activity of DIAP1 to mediate ubiquitination of dOmi.

To determine whether the interaction of dOmi-L with DIAP1 could also stimulate its auto-ubiquitination activity, we incubated DIAP1 with WT or A75P mutant dOmi-L together with Drosophila embryonic extracts and ubiquitin, and then analyzed the reaction products by western blotting with a DIAP1-specific antibody. As shown in Figure 6e, WT dOmi was able to induce robust auto-ubiquitination of DIAP1 (fourth lane, upper panel), and was itself ubiquitinated by DIAP1 (fourth lane, lower panel). In contrast, the A75P mutant dOmi$\mathrm{L}$ induced much less auto-ubiquitination of DIAP1 (fifth lane, upper panel), and was not ubiquitinated by DIAP1 (fifth lane, lower panel). Taken together, our data suggest that the interaction between dOmi and DIAP1 regulates the ubiquitination of both dOmi and DIAP1 and that this process might play important roles in the regulation of cell death and survival.

\section{Discussion}

DIAP1 is an essential regulator of developmental cell death in Drosophila, and its loss results in embryonic lethality. DIAP1 binds to and directly inhibits the proteolytic activities of the Drosophila initiator caspase Dronc (Drosophila Nedd2-like caspase) and effector caspases DCP1 and DRICE (reviewed in Meier et al., ${ }^{31}$ Ditzel and Meier, ${ }^{32}$ and Vaux and Silke ${ }^{33}$ ). DIAP1 inhibition of Dronc, DCP1, or DRICE is relieved by the IAP antagonists Reaper, Hid, Sickle, and Grim, which are cytoplasmic proteins induced during programmed cell death. ${ }^{6,10,13}$ These proteins are also essential for developmental cell death in Drosophila, which they trigger by disrupting DIAP1-caspase association and/or by inducing DIAP1's auto-ubiquitination and subsequent degradation by the proteasomal pathway. ${ }^{15-19}$

This study describes the characterization of dOmi/HtrA2, the first mitochondria-sequestered IAP antagonist in D. melanogaster. Although dOmi can antagonize DIAP1 antiapoptotic activity by directly inducing its degradation, we demonstrated that DIAP1 induces polyubiquitination of dOmi and can also antagonize dOmi-induced cell death, suggesting that DIAP1 may play a critical role to safeguard against inadvertent release of dOmi (and potentially other mitochondrial IAP-binding proteins, if they exist in Drosophila) into the cytoplasm.

Our data show that dOmi is released from mitochondria upon induction of apoptosis in cultured Drosophila S2 cells. Like its mammalian counterparts, dOmi is proteolytically processed in the mitochondria to remove its MTS. However, proteolytic maturation of dOmi generates two isoforms with distinct IBMs. Using a large-scale affinity purification process, we purified these isoforms from insect cell mitochondria and determined their exact $\mathrm{N}$-terminal amino-acid sequence by Edman degradation. This direct protein sequence analysis revealed that processing occurs at $\mathrm{G}^{74} \downarrow$ AVVS to generate the $\mathrm{dOmi}-\mathrm{L}$ isoform, and at $\mathrm{A}^{93} \downarrow \mathrm{SKMT}$ to generate the dOmi-S isoform. Our results differ from those reported recently by Challa et al., ${ }^{35}$ which predicted using the SignalP computer algorithm that processing occurs at $A^{79} \downarrow A I I Q$ and $A^{92} \downarrow A S K M$ to generate the two mature dOmi isoforms. These differences raise significant concern regarding the physiological relevance of the cell death and IAP binding results obtained with the computer-predicted mature dOmi isoforms. ${ }^{35}$ Indeed, in contrast to the previous results which demonstrated selective binding only to the BIR2 domain, ${ }^{35}$ our biochemical data show that the two dOmi isoforms bind to the BIR1 and BIR2 domains of DIAP1, but with higher affinity to the BIR2 domain. Furthermore, in contrast to the results of Challa et al., ${ }^{35}$ which reported that dOmi failed to induce or suppress DIAP1 autoubiquitination upon binding to its BIR2 domain, our data show clearly that dOmi stimulates the ubiquitination activity of DIAP1 (Figure 6c-e). These different results are clearly due to differences between the IBMs of our dOmi isoforms and the IBMs of the computer-predicted isoforms used in the previous study. ${ }^{35}$

Our findings suggest that the ability of dOmi to bind to the BIR1 and BIR2 domains of DIAP1 may have an impact on the caspase-inhibitory activities of these two domains. Since binding of the initiator caspase Dronc and IAP antagonists to the BIR2 domain of DIAP1 has been shown to be mutually exclusive, ${ }^{6}$ we predict that dOmi functions like other IAP antagonists to displace Dronc from DIAP1, thereby preventing DIAP1-mediated targeting of Dronc for ubiquitination. The effect of dOmi on DIAP1 extends to the BIR1 domain of DIAP1, which functions to inhibit the effector caspases DCP1 and DRICE. Our results demonstrate that binding of dOmi to the BIR1 or BIR2 domains of DIAP1 facilitates dOmi-mediated cleavage of the linker region between these domains and further degradation of the BIR1 domain, resulting in the liberation of DCP1 and DRICE from DIAP1 inhibition (Figure 5). This dOmi-mediated cleavage might bypass the need for ubiquitination and degradation of DIAP1 by the proteasomal pathway in the induction of apoptosis. Following displacement of DIAP1 from effector caspases by dOmi, this mechanism might thwart caspase-DIAP1 re-association, and commit the cell to apoptosis. Alternatively, dOmi-mediated degradation of DIAP1 might allow lower concentrations of IAP antagonists to induce apoptosis, which is consistent with recent observations that mitochondrial permeabilization enhances killing of Drosophila cells by IAP antagonists. ${ }^{5}$ Since mouse genetic data indicate that Omi does not play a key role in the developmental cell death pathway, ${ }^{29,30}$ we speculate that dOmi may also not be essential for developmental cell death in Drosophila. Nevertheless, the intrinsic ability of dOmi to bind to DIAP1 and to antagonize its survival activity by directly degrading it or inducing its auto-ubiquitination might tip the balance toward cell death after mitochondrial permeabilization and release of dOmi into the cytosol.

Although the biochemical data suggest that DIAP1 could interact with dOmi, it is not clear whether this interaction plays any physiological role in vivo. One possibility is that this interaction might serve as a protective mechanism to safeguard against an inadvertent release of dOmi into the cytoplasm under mitochondrial stress conditions. Indeed, our data demonstrated that overactivation of dOmi by thermal stress in Drosophila eye discs induces severe eye ablation, which could be rescued by overexpression of DIAP1. Given that DIAP1 possesses an E3 ubiquitin ligase activity, we speculate that DIAP1 association with dOmi via the IBM could stimulate its E3 ubiquitin ligase activity to promote 
polyubiquitination of dOmi and its subsequent destruction by the proteasomal pathway. Consistent with this hypothesis, our data show that DIAP1 can indeed induce polyubiquitination of dOmi in an IBM-dependent manner.

In conclusion, our data suggest that the antiapoptotic activity of IAPs in general is not restricted to direct inhibition of caspases, but may extend to protect from the caspaseindependent lethal activities of IBM-bearing proteins such as Omi. Given that many mitochondrial proteins possess $\mathrm{N}$-terminal IBMs as a result of their maturation in the mitochondria, ${ }^{37}$ our results support the hypothesis that the presence of these IBMs in mitochondrial proteins marks them for destruction if they are inadvertently released into the cytoplasm, via the E3 ubiquitin ligase activities of IAPs. The relative concentrations of IAPS and the IBM-containing proteins in the cytoplasm might, therefore, determine whether the cell would live or die. For instance, under normal stress conditions, the existing endogenous IAPs in the cytoplasm of cells could handle a small amount of released mitochondrial IBM-containing proteins. However, when the amount of an IBM-containing protein exceeds the endogenous level of IAPs, such as when dOmi is overexpressed in the mitochondria, its stress-induced release would lead to cell death. This can explain why overexpression of DIAP1 protects against cell death induced by thermal stress-activated dOmi.

\section{Materials and Methods \\ cDNA cloning and expression of recombinant proteins. The full- length dOmi cDNA clone was obtained from the IMAGE consortium (Invitrogen; GenBank $^{\mathrm{TM}}$ accession number BF499650). Constructs encoding C-terminal His $6^{-}$ tagged full-length dOmi or truncated mutants were generated by PCR using modified complementary PCR adapter primers. Plasmids encoding C-terminal GST fusion proteins containing full-length DIAP1, DIAP1-BIR1 (1-165), or DIAP1-BIR2- RING (166-438) were overexpressed in Escherichia coli strain BL21(DE3) using a modified pET21a vector made in our laboratory (the vector has C-terminal GST). Full-length XIAP, DIAP1, and DIAP2 were in vitro translated in the presence of $\left[{ }^{35}\right.$ S]methionine in reticulocyte lysates using Flag-C-pcDNA3 constructs described previously. ${ }^{20}$ Mature dOmi and its mutants were overexpressed in BL21(DE3) bacteria as $\mathrm{C}$-terminal $\mathrm{His}_{6}$-tagged proteins using a pET-21a vector. Bacterially produced proteins lack the initiator methionine, because it is removed after translation by methionyl aminopeptidase. Therefore, it is possible to produce proteins starting with different amino-acid residues.}

Large-scale production of mature dOmi in Sf9 insect cells. A baculovirus encoding full-length $\mathrm{dOmi}$ with a $\mathrm{C}$-terminal $\mathrm{His}_{6}$ tag was produced using the pVL-1393 transfer vector by standard procedures. Sf 9 cells were seeded in $20 \mathrm{~T} 175$ tissue culture flasks and then infected with the dOmi baculovirus. At $48 \mathrm{~h}$ post-infection, cells were harvested and lysed in buffer A $(20 \mathrm{mM}$ 2-[4-(2hydroxyethyl)-1-piperazinyl]-ethanesulfonic acid, $\mathrm{pH} 7.5,10 \mathrm{mM} \mathrm{KCl}, 1.5 \mathrm{mM}$ $\mathrm{MgCl}_{2}, 1 \mathrm{mM}$ ethylene diamine tetraacetic acid, $1 \mathrm{mM}$ ethylene glycol-bis $(p$ aminoethyl ether) $N, N, N^{\prime}, N^{\prime}$-tetraacetic acid, $250 \mathrm{mM}$ sucrose, $1 \mathrm{mM}$ dithiothreitol, $0.1 \mathrm{mM}$ phenylmethylsulfonyl fluoride, with protease inhibitor mix). The total lysate was centrifuged at $800 \times g$ to remove nuclei and the resultant supernatant was then centrifuged at $12500 \times g$ to obtain the mitochondrial pellet. The crude mitochondrial pellet was lysed in a buffer containing digitonin, centrifuged at $14000 \times \mathrm{g}$, and the resultant supernatant was then bound to TALON ${ }^{\mathrm{TM}}$-agarose for $1 \mathrm{~h}$ at $4{ }^{\circ} \mathrm{C}$. The bead-bound proteins were washed three times and then fractionated by SDSPAGE. The bands corresponding to the mature forms of dOmi were stained with Coomassie blue, excised from the gel, and then subjected to Edman degradation.

Additional experimental details and reagents are given in the Supplementary Information.

Acknowledgements. We thank Bruce Hay for the anti-DIAP1. We also thank the Bloomington Stock Center for Drosophila stocks. This study was supported by
NIH grants GM076176 (ESA), CA78890 (ESA), and GM50231 (JBJ), and NSF grant IOB-0416760 (JBJ). FSK is supported by NRSA award T32-CA09678.

1. Tsujimoto $\mathrm{Y}$. Cell death regulation by the Bcl-2 protein family in the mitochondria. J Cell Physiol 2003; 195: 158-167.

2. Kornbluth S, White K. Apoptosis in Drosophila: neither fish nor fowl (nor man, nor worm). J Cell Sci 2005; 118: 1779-1787.

3. Varkey J, Chen P, Jemmerson R, Abrams JM. Altered cytochrome $c$ display precedes apoptotic cell death in Drosophila. J Cell Biol 1999; 144: 701-710.

4. Igaki T, Miura M. Role of Bcl-2 family members in invertebrates. Biochim Biophys Acta 2004; 1644: 73-81.

5. Abdelwahid E, Yokokura T, Krieser RJ, Balasundaram S, Fowle WH, White K Mitochondrial disruption in Drosophila apoptosis. Dev Cell 2007; 12: 793-806.

6. Chai J, Yan N, Huh JR, Wu JW, Li W, Hay BA et al. Molecular mechanism of Reaper-GrimHid-mediated suppression of DIAP1-dependent Dronc ubiquitination. Nat Struct Biol 2003 . 10: 892-898.

7. Christich A, Kauppila S, Chen P, Sogame N, Ho SI, Abrams JM. The damage-responsive Drosophila gene sickle encodes a novel IAP binding protein similar to but distinct from reaper, grim, and hid. Curr Biol 2002; 12: 137-140.

8. Srinivasula SM, Datta P, Kobayashi M, Wu JW, Fujioka M, Hegde R et al. Sickle, a novel Drosophila death gene in the reaper/hid/grim region, encodes an IAP-inhibitory protein. Curr Biol 2002; 12: 125-130.

9. Wing JP, Karres JS, Ogdahl JL, Zhou L, Schwartz LM, Nambu JR. Drosophila sickle is a novel grim-reaper cell death activator. Curr Biol 2002; 12: 131-135.

10. Yan N, Wu JW, Chai J, Li W, Shi Y. Molecular mechanisms of DrICE inhibition by DIAP1 and removal of inhibition by Reaper, Hid and Grim. Nat Struct Mol Biol 2004; 11: 420-428.

11. Vaux DL, Silke J. Mammalian mitochondrial IAP binding proteins. Biochem Biophys Res Commun 2003; 304: 499-504.

12. Shi Y. A conserved tetrapeptide motif: potentiating apoptosis through IAP-binding 1. Cell Death Differ 2002; 9: 93-95.

13. Tenev T, Ditzel M, Zachariou A, Meier P. The antiapoptotic activity of insect IAPs requires activation by an evolutionarily conserved mechanism. Cell Death Differ 2007; 14: 1191-1201.

14. Tenev $T$, Zachariou A, Wilson R, Ditzel M, Meier P. IAPs are functionally non-equivalent and regulate effector caspases through distinct mechanisms. Nat Cell Biol 2005; 7: 70-77.

15. Hays R, Wickline L, Cagan R. Morgue mediates apoptosis in the Drosophila melanogaster retina by promoting degradation of DIAP1. Nat Cell Biol 2002; 4: 425-431.

16. Wilson R, Goyal L, Ditzel M, Zachariou A, Baker DA, Agapite J et al. The DIAP1 RING finger mediates ubiquitination of Dronc and is indispensable for regulating apoptosis. Nat Cell Biol 2002; 4: 445-450

17. Yoo SJ, Huh JR, Muro I, Yu H, Wang L, Wang SL et al. Hid, Rpr and Grim negatively regulate DIAP1 levels through distinct mechanisms. Nat Cell Biol 2002; 4: 416-424.

18. Ryoo HD, Bergmann A, Gonen H, Ciechanover A, Steller H. Regulation of Drosophila IAP1 degradation and apoptosis by reaper and ubcD1. Nat Cell Biol 2002; 4: 432-438.

19. Holley CL, Olson MR, Colon-Ramos DA, Kornbluth S. Reaper eliminates IAP proteins through stimulated IAP degradation and generalized translational inhibition. Nat Cell Biol 2002; 4: 439-444.

20. Hegde R, Srinivasula SM, Zhang Z, Wassell R, Mukattash R, Cilenti L et al. Identification of Omi/HtrA2 as a mitochondrial apoptotic serine protease that disrupts inhibitor of apoptosis protein-caspase interaction. J Biol Chem 2002; 277: 432-438.

21. Suzuki $Y$, Imai $Y$, Nakayama H, Takahashi K, Takio K, Takahashi R. A serine protease, $\mathrm{HtrA} 2$, is released from the mitochondria and interacts with XIAP, inducing cell death. Mol Cell 2001; 8: 613-621.

22. Verhagen AM, Silke J, Ekert PG, Pakusch M, Kaufmann H, Connolly LM et al. HtrA2 promotes cell death through its serine protease activity and its ability to antagonize inhibitor of apoptosis proteins. J Biol Chem 2002; 277: 445-454.

23. Martins LM, laccarino I, Tenev T, Gschmeissner S, Totty NF, Lemoine NR et al. The serine protease $\mathrm{Omi} / \mathrm{HtrA} 2$ regulates apoptosis by binding XIAP through a reaper-like motif. J Biol Chem 2002; 277: 439-444.

24. Chai J, Du C, Wu JW, Kyin S, Wang X, Shi Y. Structural and biochemical basis of apoptotic activation by Smac/DIABLO. Nature 2000; 406: 855-862.

25. Srinivasula SM, Datta $P$, Fan XJ, Fernandes-Alnemri T, Huang Z, Alnemri ES. Molecular determinants of the caspase-promoting activity of Smac/DIABLO and its role in the death receptor pathway. J Biol Chem 2000; 275: 36152-36157.

26. Srinivasula SM, Gupta S, Datta P, Zhang Z, Hegde R, Cheong N et al. Inhibitor of apoptosis proteins are substrates for the mitochondrial serine protease Omi/HtrA2. J Biol Chem 2003 278: 31469-31472.

27. Yang QH, Church-Hajduk R, Ren J, Newton ML, Du C. Omi/HtrA2 catalytic cleavage of inhibitor of apoptosis (IAP) irreversibly inactivates IAPs and facilitates caspase activity in apoptosis 58. Genes Dev 2003; 17: 1487-1496.

28. Suzuki Y, Takahashi-Niki K, Akagi T, Hashikawa T, Takahashi R. Mitochondrial protease Omi/HtrA2 enhances caspase activation through multiple pathways. Cell Death Differ 2004; 11: 208-216.

29. Martins LM, Morrison A, Klupsch K, Fedele V, Moisoi N, Teismann P et al. Neuroprotective role of the Reaper-related serine protease $\mathrm{HtrA} 2 / \mathrm{Omi}$ revealed by targeted deletion in mice. Mol Cell Biol 2004; 24: 9848-9862. 
30. Jones JM, Datta P, Srinivasula SM, Ji W, Gupta S, Zhang Z et al. Loss of Omi mitochondria protease activity causes the neuromuscular disorder of mnd2 mutant mice. Nature 2003 425: 721-727.

31. Meier P, Finch A, Evan G. Apoptosis in development. Nature 2000; 407: 796-801.

32. Ditzel M, Meier P. Ubiquitylation in apoptosis: DIAP1's (N-)en(d)igma. Cell Death Diffe 2005; 12: 1208-1212.

33. Vaux DL, Silke J. IAPs RINGs and ubiquitylation. Nat Rev Mol Cell Biol 2005; 6: 287-297.

34. Igaki T, Suzuki Y, Tokushige N, Aonuma H, Takahashi R, Miura M. Evolution of mitochondrial cell death pathway: proapoptotic role of $\mathrm{HtrA2} / \mathrm{Omi}$ in Drosophila. Biochem Biophys Res Commun 2007; 356: 993-997.

35. Challa M, Malladi S, Pellock BJ, Dresnek D, Varadarajan S, Yin YW et al. Drosophila Omi, a mitochondrial-localized IAP antagonist and proapoptotic serine protease. EMBO J 2007; 26: 3144-3156.

36. Scott FL, Denault JB, Riedl SJ, Shin H, Renatus M, Salvesen GS. XIAP inhibits caspase- 3 and -7 using two binding sites: evolutionarily conserved mechanism of IAPs. EMBO J2005; 24: $645-655$
37. Verhagen AM, Kratina TK, Hawkins CJ, Silke J, Ekert PG, Vaux DL. Identification of mammalian mitochondrial proteins that interact with IAPs via N-terminal IAP binding motifs. Cell Death Differ 2007; 14: 348-357.

38. Ditzel M, Wilson R, Tenev T, Zachariou A, Paul A, Deas E et al. Degradation of DIAP1 by the $\mathrm{N}$-end rule pathway is essential for regulating apoptosis. Nat Cell Biol 2003; 5: 467-473.

39. Martins LM, Turk BE, Cowling V, Borg A, Jarrell ET, Cantley LC et al. Binding specificity and regulation of the serine protease and PDZ domains of HtrA2/Omi. J Biol Chem 2003; 278: 49417-49427.

40. Li W, Srinivasula SM, Chai J, Li P, Wu JW, Zhang Z et al. Structural insights into the proapoptotic function of mitochondrial serine protease HtrA2/Omi. Nat Struct Biol 2002; 22: 22.

41. Plun-Favreau H, Klupsch K, Moisoi N, Gandhi S, Kjaer S, Frith D et al. The mitochondrial protease $\mathrm{Htr} \mathrm{A} 2$ is regulated by Parkinson's disease-associated kinase PINK1. Nat Cell Biol 2007; 9: 1243-1252.

42. Alnemri ES. HtrA2 and Parkinson's disease: think PINK? Nat Cell Biol 2007: 9: 1227-1229.

43. Hay BA, Maile R, Rubin GM. P element insertion-dependent gene activation in the Drosophila eye. Proc Natl Acad Sci USA 1997; 94: 5195-5200.

Supplementary Information accompanies the paper on Cell Death and Differentiation website (http://www.nature.com/cdd) 